\title{
Quantum Mechanichs Theory in Time Independent
}

\author{
Mohamad Alatawi \\ School of Art and Science \\ Clark Atlanta University \\ Atlanta, GA, 30314, USA
}

\begin{abstract}
The essential system of time-subordinate quantum-mechanical strategies for sub-atomic progression counts is portrayed. The focal issue tended to by computational strategies is a discrete portrayal of stage space. In established mechanics, stage space is spoken to by an arrangement of focuses while in quantum mechanics it is spoken to by a discrete Hilbert space. The discretization portrayed in this paper depends on collocation. Uncommon instances of this strategy incorporate the discrete variable portrayal and the Fourier technique. The Fourier strategy can speak to a framework in stage space with the proficiency of one inspecting point for each unit volume of stage space h, so that, with the best possible decision of the underlying wave work, the exponential union is gotten in connection to the quantity of testing focuses. The numerical productivity of the Fourier strategy prompts the conclusion that computational exertion scales semi linearly with the volume in the stage space involved by the sub-atomic framework. Strategies for time spread are portrayed for timeward and time-autonomous Hamiltonians. The time-free methodologies depend on a polynomial extension of the development administrator. Two of these methodologies, the Chebyshev engendering, and the Lenclos repeat, are likewise looked at. Techniques to acquire the Raman spectra specifically by utilizing the Chebyshev proliferation strategy appear. For time-subordinate issues unitary brief time propagators are depicted: the second-arrange differencing and the split administrator. The thought of every one of these strategies has prompted scaling laws of calculation. The conclusion from such scaling laws is that, for recreations of complex atomic frameworks, estimate strategies must be utilized which diminish the dimensionality of the issue.
\end{abstract}

\section{General Terms}

Time-Independent in Quantum Mechanics Theory

\section{Keywords}

Operator Method, Quantum Mechanics, Perturbation Theory, Time- Independent, and Time- Dependent

\section{INTRODUCTION}

Compound change is achieved by the movement of electrons and cores inside responding particles. The depiction of this inward movement is the subject of sub-atomic elements. Hypothetical advances in the field have been connected to the capacity to ascertain from first standards the fundamental subatomic experience[1]. This paper is worried about techniques for computation, especially those of the arrangement of the time-subordinate Schrödinger condition, applying the outcomes to the numerical reenactment of first compound occasions. The motivation behind this paper is to set rules for building, as precisely as could reasonably be expected, counts that are gone for increasing itemized knowledge into the subatomic framework and that fill in as benchmarks for other more inexact strategies. All computation techniques scale in the extent to the volume of stage space that the sub-atomic experience involves. Subsequently, stage space is a shared factor by which distinctive strategies for count can be looked at, and the plausibility of the computation evaluated. A standout amongst vital issues is the versatility and adaptability of the technique. Both these issues will be tended to by a portrayal of the major strides in time-subordinate quantummechanical figurings. As of late atomic progression has been extraordinary steps [2]. I The field has experienced a change from roundabout mass sort trials to coordinate investigations that review the rudimentary procedure without impedance of elective procedures. This segregation of essential substance experiences has propelled a theoretical understanding given first standards, i.e., the crucial conditions of movement. Disengagement of the essential occasion can be accomplished either in space or in time [3]. For gas-stage experiences or gas-surface experiences seclusion is accomplished by highvacuum methods. The analyses are by, and largely given the seeded supersonic atomic shaft. 2 In such an investigation atoms in the shaft develop at low temperatures which empower control of the underlying atomic state. With crossed-bar geometries, an immediate investigation of bimolecular responses is conceivable. 3-5 When the subatomic pillar is crossed with a laser shaft, point by point investigations of unimolecular separation can be made, specifically the separation of van der Waals molecules.6-12 Colliding the bar with a strong surface permits the examination of fundamental gas - surface experiences. The study of primary responses in the arrangement, spatial detachment is impractical. As of late grew new ultrafast laser beats in the femtosecond administration permit a period disconnection in which the necessary procedure can be examined before the encompassing dissolvable atoms can interfere[4] .A vital qualification exists amongst displaying and recreating an atomic framework. The reason for demonstrating is to pick up understanding into the component of sub-atomic marvels. A straightforward estimation indicating a conceivable hypothetical understanding is sufficient. The point of reproduction is a quantitative examination of hypothesis to try. The likelihood of reproduction in sub-atomic elements is the aftereffect of both the trial segregation of marvels and the fast change in PCs. In this present work, accentuation is on recreation strategies, despite the fact that the same hypothetical devices can serve to develop models also.

On the premise of first standards, there are four major hypothetical strategies for portraying and mimicking the basic dynamical occasion: (a) the traditional direction strategy, (b) the semiclassical technique, (c) the quantum coupled channel technique, and (d) the quantum time-subordinate technique. These fundamental techniques are likewise the establishment of the immense estimate tech* niques that have been created in hypothetical science. The working speculation in applying 
these techniques to atomic elements recreations is the BornOppenheimer estimate which permits division of the electronic from the atomic movement[5]. The theory prompts the potential vitality surface on which the concoction occasion happens. A speculation considering synchronous movement on more than one potential vitality surface is typically satisfactory for situations where the basic adiabatic BornOppenheimer guess separates. 30 The most generally utilized strategy is the established direction approach[6]. In this treatment, the movement of the cores on the BornOppenheimer potential surface is unraveled by utilizing Newton's conditions of movement. Once the potential vitality surface is known, the strategy can be effortlessly actualized. Beginning from a known introductory design, the translation of the outcomes is necessary. The strategy has been connected to a substantial number of frameworks portraying segregated experiences. The traditional direction strategy has been stretched out to forms in a dense stage by utilizing the summed up Langevin condition. It permits the depiction of frameworks in contact with a warm shower[7]. Despite its prosperity the established direction strategy has real issues. A point by point traditional direction reenactment, with full last state determination, requires broad examining in stage space which turns out to be greatly costly in calculation time. This downside is particularly huge when uncommon or moderate concoction occasions are considered. Another weakness has been found for vast atomic frameworks when the beginning of tumult is come to at low excitations, making the meaning of typical modes incomprehensible[8].Nevertheless, these downsides are minor contrasted with the way that the compound movement is quantum mechanical in nature. Quantum impacts, such as burrowing or the impact of zeropoint movement, are critical for essential concoction experiences" and speak to an inalienable obstruction to the direction approach which may prompt subjectively extraordinary outcomes. Semiclassical techniques mean to revise the defects in the traditional portrayal by including an essential quantum impacts while keeping up the straightforward usage of established mechanics[9]. The justification behind these techniques is that in light of the fact that the cores are generally substantial, quantum impacts can be dealt with as rectifications to the permanently established movement. The most created semiclassical dynamical technique is the Gaussian wave-bundle approximation[10] In this strategy the quantum wave parcel is obliged to be of a Gaussian shape. The Gaussian guess strategy has been broadly utilized as a part of numerous applications with excellent achievement. By the by, at this stage, the semiclassical strategy is an exceptionally fragile apparatus. Every framework requires a unique usage, and the legitimacy of the estimation is not known. The primary trouble in correct quantum-mechanical figurings is their nonlocal character which implies that a worldwide synchronous depiction of all the atomic stage space is required. The most develop quantum-mechanical approach is the stationary coupledchannel approach (CC), which depends on an answer to the stationary scrambling condition. 73 The technique has been connected to numerous dynamical issues, for example, receptive scattering[11], surface scattering,[12] and that's only the tip of the iceberg. In any case, in spite of broad exertion put into working out the approach, progress in reenacting sensible frameworks has been moderate. Just now, 10 years after the principal receptive disseminating result for the $\mathrm{H}+$ $\mathrm{H} 2$ framework, are comes about rising for the isotopic simple $\mathrm{D}+\mathrm{H} 2.83$ The trouble comes from the computational exertion of the technique, which scales as the quantity of channels cubed. Additionally, the strategy is hard to actualize fundamentally in light of the fact that it is a limit esteem issue which requires an uncommon treatment for every framework considered.

\section{TIME INDEPENDENT}

The time-subordinate quantum-mechanical techniques depend on the arrangement of the time-subordinate Schrödinger condition

$i \hbar \frac{\partial \psi}{\partial t}=\hat{\boldsymbol{H}} \psi$

For some environmental circumstances, time-subordinate techniques have they possess preferences. Since they are starting quality issues, the strategies are anything but difficult to execute. This implies likewise that just a single segment of the S network is computed. Specifically, the time-dependent picture empowers a basic treatment of continua and the revision issue in receptive disseminating. Other than these specific points of interest, time-subordinate strategies prompt a superior understanding of the physical component of discourse. The time variable likewise empowers a depiction of remotely determined frameworks, by presenting timesubordinate Hamiltonians. These frameworks incorporate particles subject to strong laser fields or in contact with stochastic showers. Correct time-subordinate strategies were eased back to create. At first, they were acquired by growing the state on a premise set, diagonalizing the Hamiltonian framework in this set, and utilizing the eigenvalues and eigenfunctions to proliferate in time. The numerical cost of diagonalization scales as the quantity of development capacities cubed $(\mathrm{O}(\mathrm{N} 3))$. Despite the fact that diagonalization strategies are conceivably more proficient for little straightforward model issues, given their quick computational scaling properties, they must be traded for the recreation of reasonable bigger frameworks. This was the inspiration for investigating new strategies with slower scaling properties. The initial takeoff from the quick scaling confinement was presented in crafted by McCullough and Wyatt,84 who utilized a limited distinction conspire joined with a verifiable propagator. The technique scales as $\mathrm{O}(\mathrm{N} 2)$, where $\mathrm{N}$ is the quantity of network focuses (spatial focuses used to speak to the framework). The limited distinction has been enhanced by Askar utilizing the express second-arrange differencing propagator[13] where the strategy scales as $\mathrm{O}(\mathrm{N})$ vergence rate as for framework size of limited differencing limits its utilization to just subjective issues.

The presentation of the Fourier method has tackled the issue of precision. The aftereffects of the computation join exponentially concerning lattice measure. The strategy is additionally substantially effective because of the quick Fourier change (FFT) calculation, which scales as $\mathrm{O}(\mathrm{N} \log \mathrm{m}$. Moreover, with the utilization of present-day PC design, the scaling can be diminished to $\mathrm{O}(\log \mathrm{m}$. The precision and proficiency of the Fourier strategy have made it the main case by which other calculational strategies can be looked at.

The present paper focuses on correct time-subordinate quantum-mechanical strategies. Surmised semiclassical timesubordinate techniques which have been widely considered by Heller and coworkers won't be portrayed here. The first contemplations prompting the advancement of correct timesubordinate techniques are displayed. Points of interest are described when they have significance for the general picture or when they have not been distributed beforehand, for 
example, the computation of a range utilizing the Chebychev propagator. As opposed to giving an entire survey, the accentuation is on general standards and the relationship to different strategies for the count. Extraordinary consideration is given to the development of computational scaling laws. Extrapolating on the present to a great degree fast development of the field, it is normal that time-subordinate quantum-mechanical strategies will turn into an ordinary device in physical science of both the experimentalist and the scholar. This work is pointed in this manner at opening the "black box" of the strategy and to reveal insight into the primary plan.

\section{THE USAGE OF THE TIME- SUBORDINATE STRATEGY}

The usage of the time-subordinate strategy can be isolated into five stages: (a) portrayal; (b) start; (c) Hamiltonian operation; (d) time engendering; (e) investigation of results. Segment Il is given to the portrayal of stages a-c. Segment Ill depicts steps $d$ and e. Segment IV manages current applications and the TDSCF estimation, and segment V is a review.

\subsection{Portrayal}

Stage Space and Hilbert Space. Our comprehension of natural wonders depends intensely on the possibility of congruity. Specifically, the laws of mechanics are characterized by persistent capacities. The possibility that neighborhood properties of a ceaseless capacity decide its standard character is the wellspring of the outstanding irritation approach. A similar idea is the premise of the computational methodologies in which a persistent capacity can be spoken to by an arrangement of testing focuses. A numerical approach is therefore ruled by the rate of joining of a guess given a limited arrangement of examining focuses. The proficiency of a computational plan can be judged by the convergence speed regarding the quantity of portrayal esteems.

In atomic progression, two important ideas must be spoken to: the condition of the framework and the administrators that speak to observables of the framework. There is a remarkable contrast in the way sub-atomic progression is talked to in established and in quantum mechanics, stage space being the shared factor to which a correlation can be made. In established mechanics, a point in stage space speaks to the condition of the dynamical framework[14]. A direction beginning from this point portrays the developmental progression of the framework through time. The advancement is a sign of the established conditions of movement and the underlying conditions. Information of position. In quantum mechanics, the base volume in which a robust framework can be restricted in stage space is $\mathrm{hD}$ (h is Planck's consistent and $\mathrm{D}$ the quantity of degrees of flexibility). This implies a discrete portrayal is conceivable with no less than one point for each unit volume h.D. The central nonlocal character of a quantum-mechanical framework represents the issue that the progression of a framework can't be limited to a limited volume in stage space; just its lower bound can be found out, the base esteem $\mathrm{hD}$. This is the fundamental wellspring of mistake in the limited estimate of a quantum framework.To condense, in traditional mechanics the nearby character empowers the movement to be kept to an upper limit surface in stage space. The trouble emerges given the utilization of a nearby portrayal in which a solitary point is not a satisfactory portrayal of a little neighboring volume in phase space. In quantum mechanics, then again, a strict lower limit is set by the base confinement volume P. An upper limit surface in stage space can't be entirely characterized in light of the nonlocal character of the hypothesis. The limited portrayal of stage space in quantum mechanics is the premise of the computational techniques used to understand the quantummechanical conditions of movement. The state of the unit volume component is self-assertive. This reality can be used to enhance the portrayal of a specific issue. Figure 1 shows a rectangular-formed unit volume utilized as a part of the Fourier technique depicted underneath. The development of a quantum-mechanical portrayal of stage space is firmly associated with the portrayal of the wave work in Hilbert space. In the following segment, the likelihood of precisely speaking to stage space in quantum mechanics with one point for each unit volume will be illustrated. The hypothesis of Gaussian joining at that point guarantees that the vectors gn are orthogonal. The orthogonality can be utilized to explain

$$
\begin{aligned}
& a_{n}=\sum_{j=1}^{N} f\left(x_{j}\right) g_{n}\left(x_{j}\right) \\
& f(x) \approx \sum_{j=1}^{N} \frac{f\left(x_{j}\right) g_{N}(x)}{\left(x-x_{j}\right) g_{N}{ }^{\prime}\left(x_{j}\right)}
\end{aligned}
$$

Where the examining focuses $\mathrm{xj}$ are zeros of $\mathrm{gN}(\mathrm{x})$, and $\mathrm{gN}$ can be any persistent capacity with $\mathrm{N}$ zeros. This recipe can be utilized as a reason for ascertaining subordinates on the framework focuses. The utilization of this equation scales as .O(N2) for $\mathrm{N}$ subsidiaries on all network focuses. This can be enhanced by utilizing the fractional summation strategy for Lanczos 1SS to acquire scaling as $\mathrm{O}(\mathrm{N})$

\subsection{Time Propagation}

Once the answer for speaking to the wave work and the Hamiltonian operation has been discovered, the issue of proliferating the wave work must be tended to. Formally the time-subordinate Schrödinger condition has the arrangement Before evaluating the current answers for these challenges, one can consider the confinements forced on the spread when-vitality vulnerability rule. These restrictions are normal to all proliferation plans and in this manner, fill in as a shared factor for correlation. In similarity to the force facilitate stage space, the time-vitality stage space can be considered. The volume of this stage space in units of $h$ decides the base number of focuses expected to engender the framework in time interims at with vitality run $\mathrm{AE}$. For each discrete portrayal, one can evaluate the scope of eigenvalues of the Hamiltonian administrator

$\mathrm{AE}=\mathrm{E} \max -\mathrm{E} \mathrm{mm}$. For instance, in the Fourier portrayal, the range can be assessed by including the upper and lower limits of the active and potential vitality spoke to on the lattice

$E_{\max }=\mathrm{v} \max +\mathrm{K} \max$

Once the answer for speaking to the wave work and the Hamiltonian operation has been discovered, the issue of proliferating with this definition, the development of the wave work V can be approximated as

$\psi(t) \approx e^{(-i / \hbar)\left((1 / 2) \Delta E+V_{\min }\right) t} \sum_{n=0}^{N} a_{n}\left[\frac{\Delta E t}{2 \hbar}\right] \Phi_{n}\left[-i \hat{\boldsymbol{H}}_{\mathrm{norm}}\right] \psi(0)$ 
This is evaluated by using the recursion relation of the Chebyshev polynomials

$$
\phi_{n+1}=-2 i \hat{\boldsymbol{H}}_{\text {norm }} \phi_{n}
$$

A standout amongst the most vital parts of the Chebychev engendering plan is that the blunder is consistently appropriated over all the scope of eigenvalues. Numerical cases have demonstrated that a reasonable calculation can be built affirming all the clear expectations. One disadvantage of a common advancement technique is that middle of the road comes about, which may convey much data, are not acquired. One approach to beat this issue is to part the proliferation into littler interims. The practical lower breaking point of the Chebychev extension is 40 terms. The reason is that the additional terms above $\mathrm{n}=\mathrm{a}$ which is expected to merge the aggregate start to rule, making a guess wasteful. For an understanding of transitional outcomes, this time interim is enough. However, numerous applications require the computation of relationship capacities at short interims. The Chebychev polynomial operations,

$$
=\Phi_{n}\left(-i \overline{\boldsymbol{H}}_{\text {norm }}\right) \psi(0) \text {, }
$$

Which requires the greater part of the estimation exertion, is time free? This implies the development coefficients a can be recalculated for some middle of the road times. For instance, when the relationship where Hnorm is the upper state standardized Hamiltonian, and the new extension coefficients bn are the half Fourier changes of the Bessel capacities

\section{CONCLUSION}

Time-subordinate strategies have many focal points in the investigation of atomic components. The capacity to survey previews of the wave work in middle phases of the procedure encourages distinguishing proof of an atomic system. Concentrates on these lines have been given to the procedure of reverberation rot. The frameworks contemplated incorporating the separation flow of the collinear van der Waals complex of He12[15] This investigation was completed by both a correct time-subordinate technique and the TDSCF estimation. Point by point examines the separation elements of the model collinear dynamically coupled Morse oscillators have demonstrated an incredible assortment of separation lifetimes for resonances with nearly the same Receptive dissipating issues are the slowest to have been created. Numerous collinear responses have been examined with the favorable primary position of picking up knowledge into the point by point elements. The frameworks examined incorporate $\mathrm{H}+\mathrm{H} 2, \mathrm{~F}+\mathrm{DBr}, \mathrm{H}++\mathrm{H} 2,[16]$ and that's only the tip of the iceberg. Additionally, impact instigated separation figurings were performed. Here the timesubordinate technique was picked due to its capacity to treat various continua. Almost untouched ward counts are confined to collinear geometry. The reason is the intricacy of the organizing framework for an aggregate $\mathbf{J}$ preservation. The following curvilinear directions are not just spoken to by the Fourier technique, and other collocation strategies have still to be produced. Reproducing these responsive frameworks in six-dimensional Cartesian directions might be alluring. The straightforwardness will make up for the sparing because of the protection of precise aggregate energy. The most recent point is the reproduction of electrons in consolidated stages. The system is a TDSCF quantum - established computation where the elements of the atomic movement are dealt with traditionally. Computations were connected to an electron in a group i46 and electrons in liquid salts[17]. Advances have been likewise made in the treatment of electronic movement by static SCF computations utilizing pseudospectral strategies. Anticipating to new applications, techniques scaling as the Fourier strategy will assume a regular part. As the reproduced issue winds up noticeably bigger, the slower scaling strategies turn out to be increasingly alluring. The Fourier strategy, while permitting high precision since it depicts the nonlocal character of quantum mechanics effectively. has the slowest scaling properties. prerequisites in the present calculations, whose lifetime may well stretch out into the next PC era. The dependable guideline of PC drafters is that like clockwork another PC era is conceived, with registering power augmented by a request of size. Since computational exertion scales as the volume in stage space, this suggests the wilderness of possible issues is pushed one measurement forward each seven or eight years. The present status is that, on quick minicomputers, three-dimensional atomic issues can be performed and, on supercomputers, fourdimensional issues can be performed. For electrons, which are lighter, the present status is that six-dimensional figurings can be performed. From this, the conclusion can promptly be drawn that, even with the quick improvement of new PCs, the wilderness keeps on moving gradually. This focuses on the significance of growing great estimate techniques, rather than holding up until the year 2000, for explaining a sixdimensional sensitive dissipating issue. A similar issue can be comprehended today by suitably isolating out two subspaces by utilizing the TDSCF strategy. The benchmark capacity of specific time-subordinate strategies is assistance in increasing physical knowledge into how important the guessing procedure is. Other than the pattern of quicker machines with bigger memory, there is another pattern that can be seen today, which is the advancement of parallel machines. Since there is the farthest point to how quick a single processor can function, the positive approach to extend registering power is to interface huge numbers of them into a parallel machine. Calculations that can be parallelizable may get by into the next PC era, while others that can't die. Therefore, calculations that are mediocre on following machines may be the common ones on parallel machines. This reality requires a reconsideration of existing calculations, in light of the advancement of parallel new calculations. The Fourier strategy is exceedingly parallelizable since FFT's are. The TDSCF technique can be actualized in parallel frequently and given this may pick up outstanding quality. All in all, the blend of collocation strategies profoundly picked up by parallelizable guess calculations may be the correct approach to stand up to high-dimensional atomic flow issues later on. Time-subordinate quantum-mechanical strategies will assume a noteworthy part in future recreations as a result of their precision, adaptability, and productivity prompting more prominent knowledge into dynamical atomic procedures. 

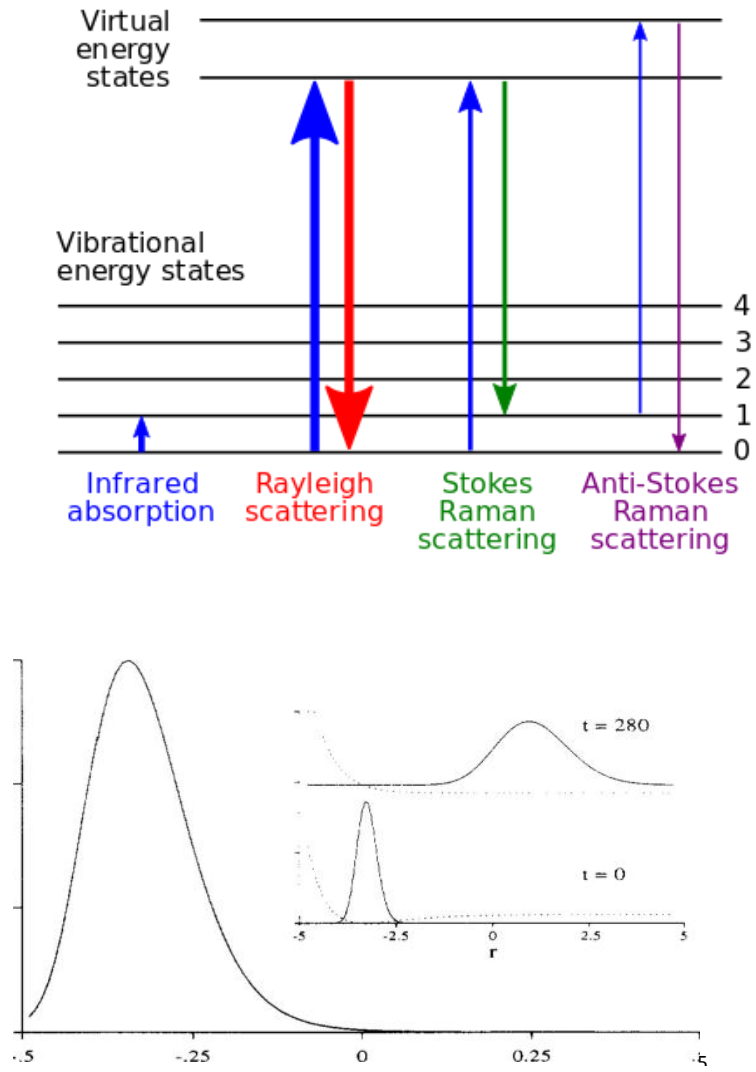

FIG1: Raman range of ground-state Morse oscillator and an energized state appalling potential. The lower board demonstrates the spectra lai012 as an element of Ace. The wide line is a consequence of the quick photodissociation on the upper shocking potential. The upper right board demonstrates the ground-state beginning state and the groundstate potential (dashed line) and the upper state wave work after 280 au of time on the horrendous potential (dashed line). The Morse potential parameters were Do $=0.174 \mathrm{au},=0.9374$ au- 1 , and mass $\mathrm{m}=911 \mathrm{au}$. The upper terrible potential parameters are DI $=0.164$ au and $B 1=0.9374$ au-1. A network of 128 focuses was utilized with $\mathrm{Ax}=0.075 \mathrm{au}$. The quantity of Chebychev coefficients in the computation was 436 .

\section{The usage of time independent strategy}

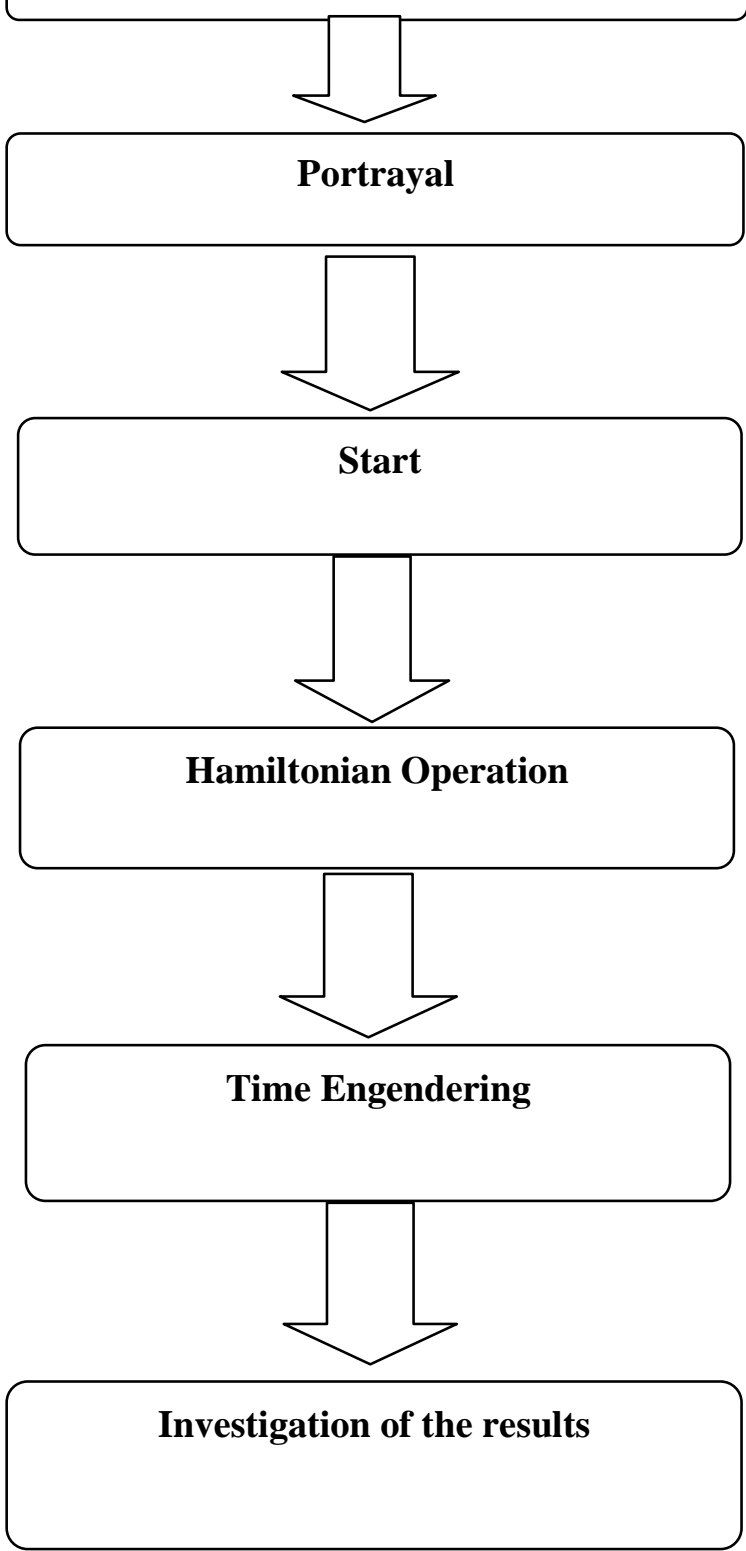

\section{REFERENCES}

[1] Hershbach, D. R. Adv. Chem. Phys. 1966, 10, 319.

[2] Beswick, J. A.; Jortner, J. Adv. Chem. Phys. 1981, 47, 363.

[3] Drolshagen, G.; Heller, E. J. Chem. Phys. Lett. 1984, 104, 129.

[4] Jackson, B.; Metiu, H. J. Chem. Phys. 1985, 82, 5707

[5] Daniel, J.; Heller, E. J. Chem. Phys. 1987, 87, 5302.

[6] Webster, F.; Light, J. C. J. Chem. Phys. 1986, 85, 4744.

[7] Christoffel, K. M.; Brumer, P. Phys. Rev. A 1986, 33, 1309.

[8] Kottler, Z.; Nitzan, A.; Kosloff, R. In Proceedings of the 19th Jerusalem Symposium; Pullman, B., Jortner, J. Eds.; Reidel: Dordrecht, Holland, 1986 
[9] Nauts, A.; Wayatt, R. E. Phys. Rev. Lett. 1983, 51, 2238.

[10] Gerber, R. B.; Buch, V.; Ratner, M. A. J. Chem. Phys. 1982, 77, 3022.

[11] Luk, T. S.; Johann, I-J,; Egger, H,; Pummer, H.; Rhods, C. K. Phys. Rev. A 1985, 32, 214.
[12] Nauts, A.; Wayatt, R. E. Phys. Rev. Lett. 1983, 51, 2238.

[13] Kosloff, R.; Kosloff, D, J. Comput. Phys. 1986, 63, 363.

[14] Bacic, Z.; Gerber, R. B.; Ratner, M. A. J. Phys. Chem. 1986, 90, 3606.

[15] Tadmor, E. SIAM J. Numer. Anal. 1986, 23. 